\title{
Impacts of Microplastics on the Soil Biophysical Environment
}

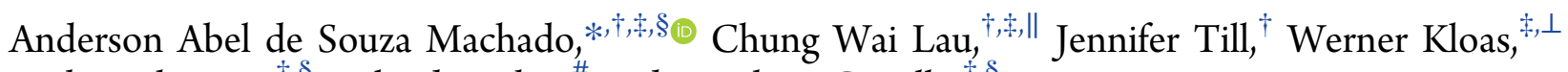 \\ Anika Lehmann, ${ }^{\dagger, \S}$ Roland Becker, ${ }^{\#}$ and Matthias C. Rillig ${ }^{\dagger, \S}$ \\ ${ }^{\dagger}$ Institute of Biology, Freie Universität Berlin, Berlin, Germany \\ ${ }^{\ddagger}$ Leibniz- Institute of Freshwater Ecology and Inland Fisheries, Berlin, Germany \\ ${ }^{\S}$ Berlin- Brandenburg Institute of Advanced Biodiversity Research, Berlin, Germany \\ "Faculty of Forestry, University of Göttingen, Göttingen, Germany \\ ${ }^{\perp}$ Faculty of Life Sciences, Humboldt-Universität zu Berlin, Berlin, Germany \\ \#Bundesanstalt für Materialforschung und-prüfung, Berlin, Germany
}

Supporting Information

\begin{abstract}
Soils are essential components of terrestrial ecosystems that experience strong pollution pressure. Microplastic contamination of soils is being increasingly documented, with potential consequences for soil biodiversity and function. Notwithstanding, data on effects of such contaminants on fundamental properties potentially impacting soil biota are lacking. The present study explores the potential of microplastics to disturb vital relationships between soil and water, as well as its consequences for soil structure and microbial function. During a 5-weeks garden experiment we exposed a loamy sand soil to environmentally relevant nominal concentrations (up to $2 \%$ ) of four common microplastic types (polyacrylic fibers, polyamide beads,
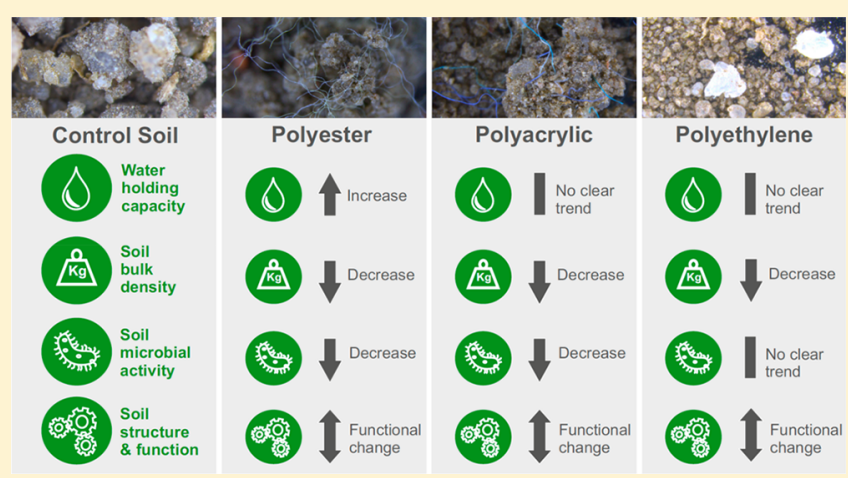

Polyethylene polyester fibers, and polyethylene fragments). Then, we measured bulk density, water holding capacity, hydraulic conductivity, soil aggregation, and microbial activity. Microplastics affected the bulk density, water holding capacity, and the functional relationship between the microbial activity and water stable aggregates. The effects are underestimated if idiosyncrasies of particle type and concentrations are neglected, suggesting that purely qualitative environmental microplastic data might be of limited value for the assessment of effects in soil. If extended to other soils and plastic types, the processes unravelled here suggest that microplastics are relevant long-term anthropogenic stressors and drivers of global change in terrestrial ecosystems.
\end{abstract}

\section{INTRODUCTION}

Soils mediate a multitude of services such as carbon sequestration, biogeochemical cycling and biodiversity promotion. ${ }^{1,2}$ An important underlying engine powering most of these services is the activity of soil microbes, which catalyze many of the biogeochemical transformations that yield human societal goods, for example, increasing food security. ${ }^{3}$ In turn, the function of such microbes is strongly influenced by the physical and chemical conditions to which these organisms are exposed. For instance, the arrangement of soils into various functional aggregates, pore space configuration, and hydrological properties are known to affect microbial metabolic rates and organic matter decomposition. ${ }^{4}$ In other words, fundamental properties affecting the soil biophysical environment are important for soil processes and function. Our mechanistic understanding of the effects of emerging ecosystem stressors such as microplastics on soil functional changes lags behind comparable studies in aquatic ecosystems, ${ }^{5}$ and soil biophysical factors may be the linchpin for understanding these effects.
Microplastics represent potential threat for soil biota if contamination would cause changes on the soil habitat. Empirical calculations suggest that about $32 \%$ of all plastic produced is environmentally available in continental systems, ${ }^{6}$ and certain authors argue that soils might store more microplastic litter than oceanic basins. ${ }^{7}$ A variety of human activities and environmental sources might contribute to terrestrial contamination, ${ }^{8}$ for example. plastic mulches, ${ }^{9,10}$ contaminated water courses, ${ }^{11}$ atmospheric precipitation, ${ }^{12}$ and compost used as an agricultural amendment. ${ }^{13}$ Microplastics have been found in soils from nonurban natural reserves including mountainous and inhabited areas with a baseline level up to $0.002 \%$ of soil dry weight. ${ }^{14}$ Upon arrival at the soil surface, microplastics can be effectively incorporated to the soil matrix by bioturbation, ${ }^{15-17}$ soil management

Received: April 25, 2018

Revised: June 29, 2018

Accepted: July 27, 2018

Published: July 27, 2018 
practices, ${ }^{10}$ and water percolation, ${ }^{18}$ among other processes. ${ }^{19}$ It is currently not possible to determine accurately the final fate for this contaminant in soils. ${ }^{8}$ However, at time scales relevant for human life and pollution management, it is reasonable to assume a near-permanent and increasing microplastic terrestrial pollution. $^{20}$ In highly contaminated top soils concentrations as high as $7 \%$ of microplastic weight have been reported. ${ }^{21}$ To the best of our knowledge, the potential changes in soil biophysical properties triggered by microplastic contamination have not been studied.

The non-natural properties and persistence of microplastic terrestrial pollution might qualify these particles to be drivers of environmental change. Therefore, it is important to investigate the impacts of this contaminant on the natural relationships between soil particles and its biota. The present study examines the potential of microplastics to disturb natural biophysical properties of the soil environment. Using traditional and well-established proxies of soil health and function, we here report results on the effects on basic soil physical parameters, as well as soil structure and microbial function. We discuss the environmental significance of such novel microplastic impacts on soil properties, elaborating on the limitations of this initial assessment, and highlighting future research required for testing the possible broad consequences of the present results in a global change context.

\section{MATERIAL AND METHODS}

Our test soil was a loamy sand, collected at the experimental facilities of the Freie Universität Berlin $\left(52^{\circ} 27^{\prime} 58^{\prime \prime} \mathrm{N}\right.$, $13^{\circ} 18^{\prime} 10^{\prime \prime}$ E; Berlin, Germany) in October 2016 and stored in the greenhouse $\left(\sim 21 \pm 2{ }^{\circ} \mathrm{C}\right)$. Further properties of this soil were already reported in Supporting Information (SI) S1B and elsewhere. $^{22,23}$ In May 2017, this soil was sieved at $5 \mathrm{~mm}$ to remove gravel and large roots, and then manually disaggregated by vigorously grinding $\left(\sim 5 \mathrm{~min} \mathrm{~kg}\right.$ of soil $\left.{ }^{-1}\right)$ the soil in a metal container with a stone $(\sim 10 \mathrm{~cm}$ diameter $)$ from the same soil origin. This partially disaggregated soil was drysieved at $630 \mu \mathrm{m}$ for removal of large aggregates, and the fraction passing this sieve was used for the experiment. The selection of soil particles smaller than $630 \mu \mathrm{m}$ for the experiment aimed at increasing the likelihood of detecting changes in soil aggregation levels as assessed with the methods described below.

Microplastic Addition to Soil. Four types of microplastic particles were considered in the current experiment (Figure 1). The diversity of particle models used here aimed at a broad representation of potential mechanisms of microplastic effects on soils. Thus, the various particle models considered here increase the validity of the statements on whether microplastic particles can affect the soil properties considered. Unravelling whether polymer, particle size, or any covariate property are the responsible for the observed effects is beyond the scope of the current article, and such speculations are avoided throughout the text. The polyacrylic fibers were obtained manually cutting 100\% acrylic "Rozetti Puzzle" yarn (product number 233-01, Himalaya Co. Turkey). These fibers had an average length of $3756 \mu \mathrm{m}(\min =1260 \mu \mathrm{m}, \max =9100 \mu \mathrm{m}$, $N=47)$ and an average diameter of $18 \mu \mathrm{m}(\min =12 \mu \mathrm{m}, \max$ $=24 \mu \mathrm{m}, N=47$ ). Polyamide beads of $15-20 \mu \mathrm{m}$ diameter (product AM306010) were acquired from Good Fellow (Cambridge, United Kingdom). Microscopic inspection confirmed that those particles were reasonably spherical and had diameters with little deviation from the nominal size.

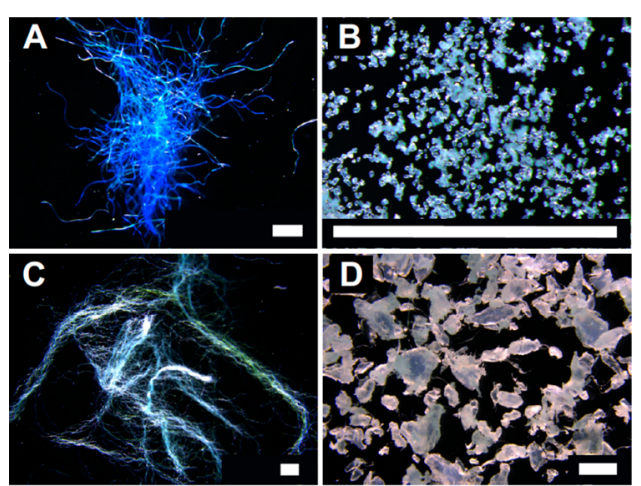

Figure 1. Four microplastic types considered in the current study. Polyacrylic fibers (A), polyamide beads (B), polyester fibers (C), and polyethylene high-density fragments $(D)$. The white bar in each panel represents $1 \mathrm{~mm}$ size.

Polyester fibers were manufactured by manually cutting $100 \%$ polyester wool "Dolphin Baby" (product number 80313, Himalaya Co. Turkey). Polyester fibers had an average length of $5000 \mu \mathrm{m}(\min =1540 \mu \mathrm{m}, \max =6300 \mu \mathrm{m}, N=47)$ and an average diameter of $8 \mu \mathrm{m}(\mathrm{min}=6 \mu \mathrm{m}, \max =10 \mu \mathrm{m}, N=47)$. Finally, polyethylene high-density fragments with an average largest dimension of $643 \mu \mathrm{m}(\min =160 \mu \mathrm{m}, \max =1200 \mu \mathrm{m}$, $N=7$ ) were fabricated by the Bundesanstalt für Materialforschung und -prüfung (Berlin, Germany) by cryo-milling pristine industrial pellets. As the self-manufactured microparticles of polyacrylic, polyester, and polyethylene presented size variability larger than $10 \mu \mathrm{m}$, their particle size distributions are presented in SI Figure S1 A. For practical reasons, we refer hereafter to the particle type by its polymer matrix or whether its physical structure was linear (fibers) or nonlinear (beads and fragments).

Each microplastic type was microwaved for $3 \mathrm{~min}$ to minimize microbial contamination and then added to the experimental soil at four nominal concentrations. The particles did not seem to be physically affected by the microwave procedure as confirmed by steromicroscope inspection. Polyacrylic and polyester fibers were tested by adding, respectively, $0.05 \%, 0.10 \%, 0.20 \%$, and $0.40 \%$ of soil dry weight in microplastic particles. The upper limit concentration was determined based on the highest concentration at which soils experienced minor changes in volume after the addition of linear microplastics. Polyamide and polyethylene microplastics were tested at $0.25 \%, 0.50 \%, 1.00 \%$, and $2.00 \%$ or nominal microplastic weight. The upper limit concentration of nonlinear microplastics was determined based on maximum microplastic contamination in which no visual changes in soil after microplastic addition could be detected, that is, these particles completely blended into the soil matrix. These contamination levels are environmentally relevant as soils up to $7 \%$ of microplastic fragments have been reported. ${ }^{21}$ The mixing of plastic and soil was performed in a glass beaker by manually stirring with a metal spoon $\sim 700 \mathrm{~g}$ of experimental soil during $10 \mathrm{~min}$. An additional control treatment was included, with no plastic addition but equivalent $10 \mathrm{~min}$ stirring.

We estimated the number of microplastic particles, surface area and volume based on average particle size and polymer density (i.e., polyacrylic $=1184 \mathrm{~kg} \mathrm{~m}^{-3}$, polyamide $=1350 \mathrm{~kg}$ $\mathrm{m}^{-3}$, polyester $=1370 \mathrm{~kg} \mathrm{~m}^{-3}$, and polyethylene high-density $=$ $970 \mathrm{~kg} \mathrm{~m}^{-3}$ ). It is important to mention that despite the fact 
that the control treatment represents no addition of experimental plastic (nominal concentration 0\%), it does not represent absolute absence of microplastics in the soil. In fact, as the soils were originally collected from an urban area, actual levels of microplastics in the control could possibly be close to the lower concentrations of microplastics added. Notwithstanding, our lower exposure levels are in the range of the highest contamination reported (see review ${ }^{8}$ ). In this sense, the lower exposure concentrations might at least represent significant shifts in the main polymer and particle type, while the higher exposure treatments represent also substantial increases in total microplastics content of the soils. Quantifications of plastics were not performed as there is no established methodology for extraction and simultaneous quali-quantitative nontargeted measurement of microplastic concentrations in soils ${ }^{24}$ and analytical approaches are currently being developed. ${ }^{25}$ Therefore, soil exposure based on the nominal concentrations will be presented hereafter.

Experimental Set up and Proxies of Soil Biophysical Environment. For each experimental replicate $70 \pm 1 \mathrm{~g}$ of soil or soil-microplastic mixture was added to black polypropylene preweighted pots of $5.5 \mathrm{~cm}$ height and $5.7 \mathrm{~cm}$ diameter (Pöppelmann GmbH, Germany). Control $(N=10)$ and microplastic treatments $(N=5)$ were then partially buried in a randomly assigned position within an area of $\sim 4 \mathrm{~m}^{2}$ (SI Figure S1 B) in the experimental garden of Freie Universität Berlin ( $52^{\circ} 27^{\prime} 25.55^{\prime \prime} \mathrm{N}, 13^{\circ} 18^{\prime} 6.28^{\prime \prime}$ E, Berlin- Germany). There were four holes in the bottom of the pots $(\sim 1 \mathrm{~cm}$ diameter) covered with mesh DuPont Plantex (DuPont de Nemours, Luxemburg) that allowed drainage, while the upper portion was open (Supporting Informaiton Figure S1B), thus allowing the experimental soils to undergo near-natural diurnal temperature and humidity variation. All the experimental units were covered with fresh above-ground biomass of locally available grass (mostly Elymus repens) and remained in the garden for $\sim 5$ weeks (29 of May to 03 of July, 2017).

Upon removal from the soil, each pot was stored at $4{ }^{\circ} \mathrm{C}$ for 4 days. Any organisms visible at the soil surface as well as germinating seedlings within the pots were removed. The general microbial activity was then assessed in $0.5 \mathrm{~g}$ of the surface of experimental soil using hydrolysis of fluorescein diacetate $(\mathrm{FDA})^{26}$ with three analytical replicates and adaptations for a 96 wells microplate reader (Tecan; Infinite M200, Männedorf, Switzerland).

The whole soil remaining structurally intact in the pot was analyzed for several established proxies of soil function regarding hydrological and structural properties. Hydraulic conductivity $(\mathrm{K})$ was measured using the flow induction with constant head method ${ }^{27}$ described in details in the SI Figure S1B. Bulk density was computed by measuring the volume of soil within the pot and soil dry weight. Soil structure was assessed by gently pushing the whole soil through a set of stacked sieves (mesh opening of $4000 \mu \mathrm{m}, 2000 \mu \mathrm{m}, 1000 \mu \mathrm{m}$, $630 \mu \mathrm{m}$ ). After recording the weight of each sieved fraction, we reconstituted the whole sample and assessed water stable aggregates in a $\sim 4.0 \mathrm{~g}$ aliquot using a wet sieving apparatus (Eijkelkamp Co., Giesbeek, The Netherlands) to evaluate soil stability against water as disintegrating force (mesh opening of $250 \mu \mathrm{m})^{28}$ while correcting for coarse matter larger than 250 $\mu \mathrm{m}$.

Statistical Inference. All results reported here were compiled in a data table provided as SI S2. All the statistical inference and data plotting was done in $\mathrm{R}$ version 3.4.4. ${ }^{29}$ The library "dplyr" ${ }^{30}$ with built-in and user-defined functions was used for data handling. Statistical inference of significant effects was tested with functions " $1 \mathrm{~m}$ " (for traditional linear models ${ }^{29}$ ), "gls" (for generalized linear models ${ }^{31}$ ), or "lmer" (for generalized linear mixed-effects models ${ }^{32}$ ), whenever appropriate. Generally, the measured end point (e.g., soil bulk density) was modeled as a function of microplastic concentration, particle type, particle number, particle volume, and particle surface area. During the determination of random and fixed structures for each linear model the functions "anova" and library "MuMIn" 33 were used for model comparisons. In all cases the significance threshold was $5 \%$ $(\alpha=0.05)$, and $p$ values presented in the main text refer to the slope of the respective fixed factor. The process of model selection resulted in several linear models. Thus, readers interested in specific model structures are encouraged to check the table with statistical inference pipeline in SI 1 (Table S1B) and the $\mathrm{R}$ script with all statistical analyses provided as Supporting Information. The libraries "ggplot2", 34 "ggthemes", 35 and "cowplot" 36 were used for data visualization.

\section{RESULTS AND DISCUSSION}

The polyacrylic, polyester, and polyethylene particles seemed intact when observed at the stereomicroscope after the 5-week period (Figure 2). These particles were incorporated into the
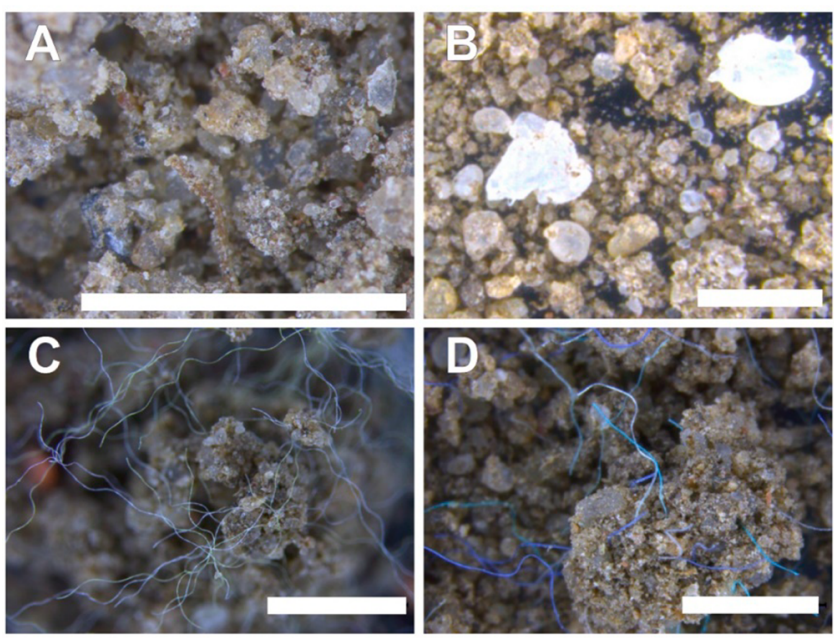

Figure 2. Integration of microplastic particles to the soil biophysical environment. Structure of control soil (A) was not visually distinct under the stereomicroscope from soil contaminated with polyamide beads (SI S1D). Polyethylene fragments (B), and polyester (C) or polyacrylic fibers (D) resulted in visually apparent soil features. The white bar in each panel represents $1 \mathrm{~mm}$ size.

soil matrix in a distinct manner, however. While polyethylene fragments interacted very loosely with other soil particles (Figure 2B), the linear shape of polyacrylic and polyester fibers formed the skeleton for larger clumps of soil. The implications of such findings are not easy to extrapolate for environmental conditions (see SI S1C). Notwithstanding, it might be reasonable that such impacts of microplastics on the formation of larger or smaller soil clumps could have consequences for soil erosion worthy of further investigation.

Most of the polyacrylic, polyester and polyethylene particles could be easily identified with the stereomicroscope. However, polyamide beads could not be conclusively distinguished from soil particles (SI Figure S1 D). As a significant degradation of 

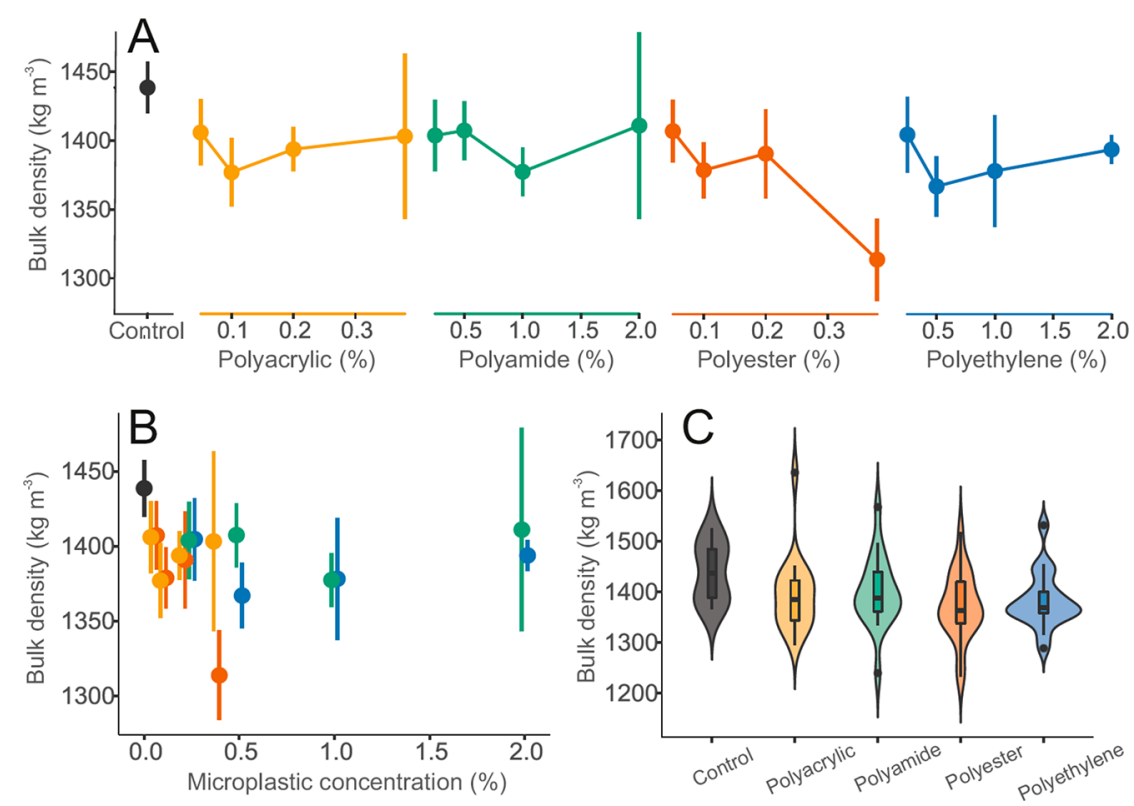

Figure 3. Effects of microplastic particles on soil bulk density. (A) Visualization of the impact of microplastics on bulk density over the range of treatment concentrations. (B) Focusing on effects of microplastic concentration irrespective of microplastic type. For A and B, data were represented by mean and standard error ( $N=5$ per microplastic treatments, 10 for controls). (C) Summary of effect range of microplastic types for bulk density combining treatment concentration. Data distribution was depicted by violin plots with median, interquartile range and $95 \%$ confidence interval overlaid. Dots represent outlying data. Microplastic types are color-coded: controls (dark gray), polyacrylic (yellow), polyamide (green), polyester (red) and polyethylene (blue) treatments (i.e., linear microplastics are in warm colors- yellow and red, nonlinear microplastics are in cold colors- blue and green).
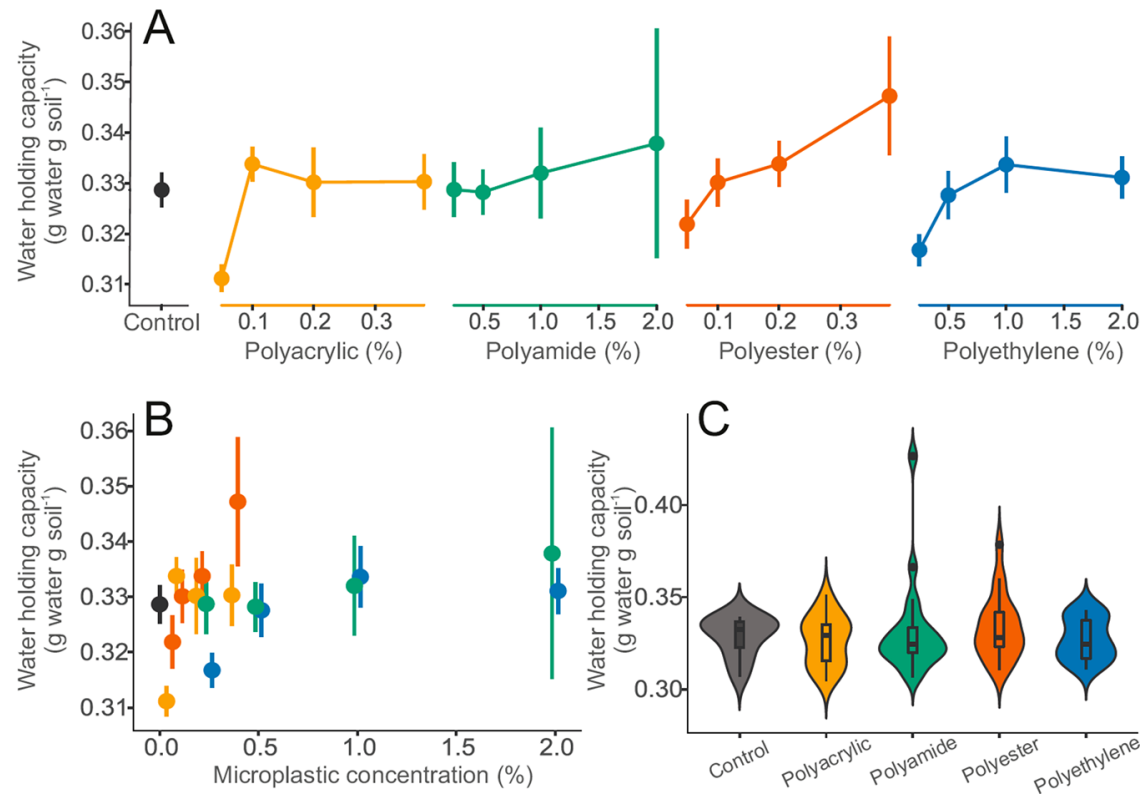

Figure 4. Effects of microplastic particles on water holding capacity. (A) Visualization of the impact of microplastics on water holding capacity over the range of treatment concentrations. (B) Focusing on effects of microplastic concentration irrespective of microplastic type. For A and B, data were represented by mean and standard error $(N=5$ per microplastic treatments, 10 for controls). (C) Summary of effect range of microplastic types for bulk density combining treatment concentration. Data distribution was depicted by violin plots with median, interquartile range and $95 \%$ confidence interval overlaid. Dots represent outlying data. Microplastic types are color-coded: controls (dark gray), polyacrylic (yellow), polyamide (green), polyester (red), and polyethylene (blue) treatments (i.e., linear microplastics are in warm colors: yellow and red, nonlinear microplastics are in cold colors: blue and green).

such polymers is unlikely under our experimental conditions, ${ }^{37}$ polyamide beads were likely effectively incorporated into the soil matrix. This calls for a critical evaluation of available literature that relies strongly on visual cues for qualiquantitative assessments of microplastics in soils without assessments of the effectiveness procedures for extraction of small microplastics.

Microplastics Effects on the Soil Biophysical Environment. All tested particles affected soil bulk density $(p<0.001$, Figure $3 \mathrm{C}$ ), and the polyester fibers were observed to cause a 

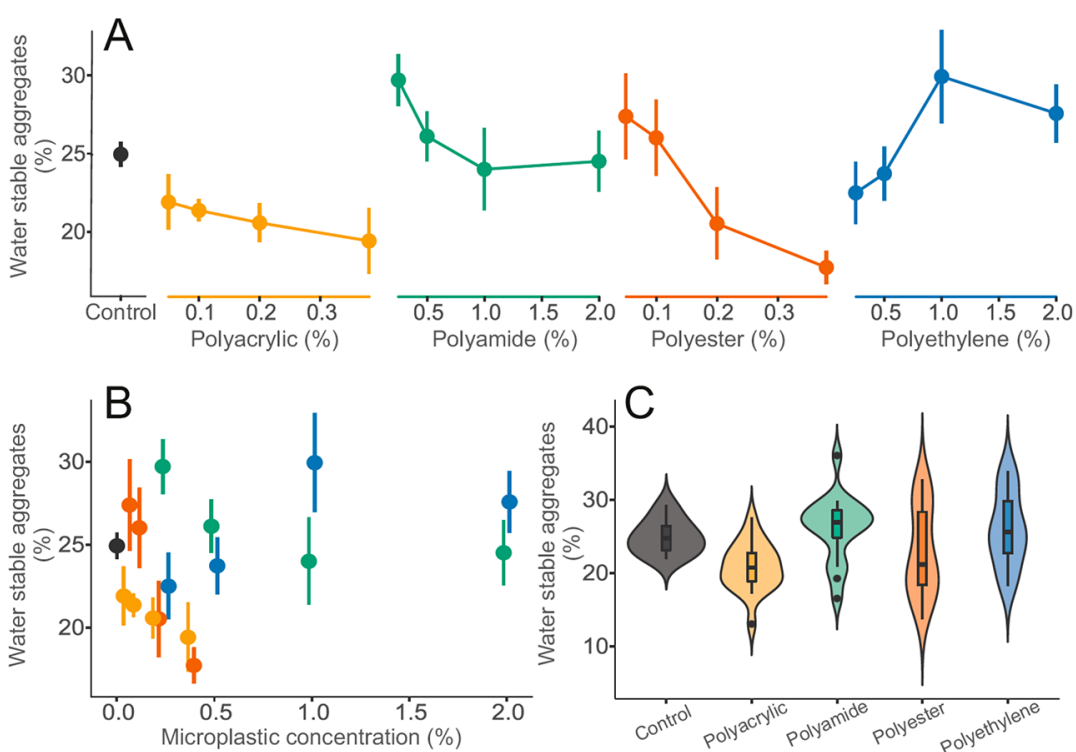

Figure 5. Effects of microplastic particles on the soil structure. (A) Visualization of the impact of microplastics on bulk density over the range of treatment concentrations. (B) Focusing on effects of microplastic concentration irrespective of microplastic type. For A and B, data were represented by mean and standard error $(N=5$ per microplastic treatments, 10 for controls). (C) Summary of effect range of microplastic types for bulk density combining treatment concentration. Data distribution was depicted by violin plots with median, interquartile range and $95 \%$ confidence interval overlaid. Dots represent outlying data. Microplastic types are color-coded: controls (dark gray), polyacrylic (yellow), polyamide (green), polyester (red), and polyethylene (blue) treatments (i.e., linear microplastics are in warm colors: yellow and red, nonlinear microplastics are in cold color: blue and green).

concentration dependent response $(F=6.32, p<0.05)$. These shifts in bulk density might be partially explained by the fact that plastics are often less dense than many natural minerals predominant in soils. Indeed, the control soils presented a bulk density of about $1439 \pm 86 \mathrm{~kg} \mathrm{~m}^{-3}$ at the end of our experiment while the densest microplastic polymer used here was the polyester $\left(\sim 1370 \mathrm{~kg} \mathrm{~m}^{-3}\right)$. There might be an additional role of microplastic type in affecting the pore space and particle interaction within the soil, however. Polyacrylic fibers and polyethylene fragments did not trigger as marked decreases in bulk density as did polyester fibers despite that these polymers present only $\sim 86 \%$ and $\sim 71 \%$ of polyester density, respectively. Within the microplastic levels tested here, the semiquantitative exposure metrics (Figure 3B, SI Figure S1E) do not reveal a statistically significant trend.

Polyester fibers also affected water holding capacity of the soil (Figure 4A). Compared to other microplastics, increasing concentrations significantly enhanced this parameter $(F=7.07$, $p<0.05)$. Under the experimental conditions tested here, none of the other particles elicited similar effects. These fibers have potential for long-distance environmental atmospheric ${ }^{12}$ and fluvial transport. ${ }^{.4}$ The modest increase in water holding capacity, if generalizable, might affect soil moisture and evapotranspiration; important phenomena for ecosystem services and feedback to the regional and global climate. ${ }^{1}$ The semiquantitative or qualitative exposure metrics, that is, microplastic levels disregarding the identity of the particle polymer, type, and mass, did not reveal any significant trend (Figure 4 B-F). Despite the noticeable changes in soil bulk density, and water holding capacity, we did not detect significant changes in hydraulic conductivity (SI S1E).

Diverse impacts of microplastics on soil structure were also observed (Figure 5). Soils contaminated with polyester fibers presented a significant decrease in water stable aggregates with increasing polyester concentrations $(F=12.04, p<0.01)$. In terms of semiquantitative exposure metrics, no significant effects were found concerning microplastic mass, particle number, surface area, or volume, if particle type was disregarded (Figure 4B, D-F). However, considering qualitative exposure metrics (Figure 5C) soils containing polyacrylic fibers displayed a significant decrease in water stable aggregates $(p<0.05)$. Moreover, multiple significant changes on soil structure after dry sieving were observed (SI S1F).

Polyester fibers caused most apparent changes in the proxies of soil biophysical parameters assessed here, for example, concentration dependent trends for effects on soil bulk density, water holding capacity, and soil structure. In the absence of conclusive scientific information to elucidate the mechanisms of such impacts, we can propose some hypotheses based on our empirical observations of the larger soil concretions. The shape of fibers might imply higher potential to change soil biophysical properties as such linear structures differ substantially from the nonlinear particles that composed the bulk of soil mass. A combination of plastic properties and particle shape means that polyester fibers were more flexible, and thus blended more effectively and homogeneously within the soil matrix than their polyacrylic counterparts. In turn, that would allow more efficient entanglement of soil particles by polyester fibers. Indeed, polyester-influenced soil concretions were generally more diverse in sizes than polyacrylic held clumps when qualitatively observed under stereomicroscope. Polyester fibers modeled the edges of soil clumps at smaller spatial scale (Figure 2C), while the edges of polyacrylicinfluenced concretions were more influenced by interaction among natural soil particles (Figure 2D). Thus, it is plausible that the ability to form soil clumps and entangle soil particles at finer spatial scales accounted for the more pronounced effects of polyester fibers used here. 

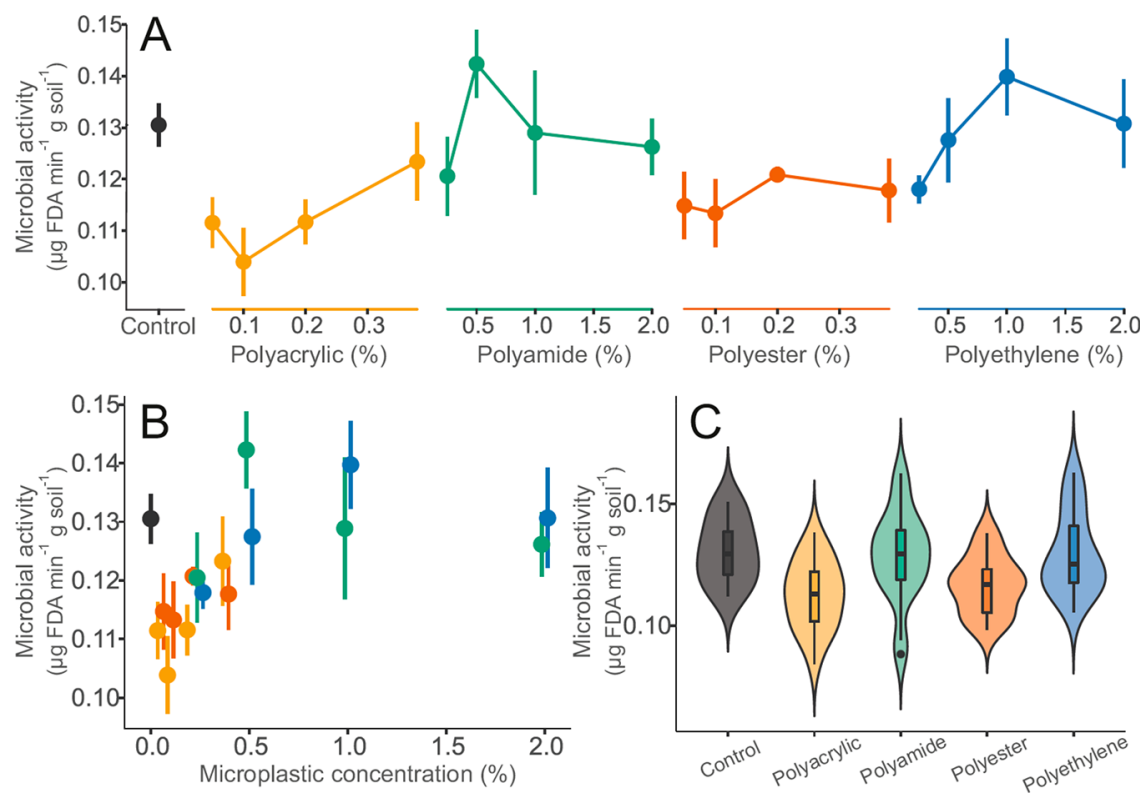

Figure 6. Effects of microplastic particles on soil microbial activity. (A) Visualization of the impact of microplastics on bulk density over the range of treatment concentrations. (B) Focusing on effects of microplastic concentration irrespective of microplastic type. For A and B, data were represented by mean and standard error $(N=5$ per microplastic treatments, 10 for controls). (C) Summary of effect range of microplastic types for bulk density combining treatment concentration. Data distribution was depicted by violin plots with median, interquartile range and $95 \%$ confidence interval overlaid. Dots represent outlying data. Microplastic types are color-coded: controls (dark gray), polyacrylic (yellow), polyamide (green), polyester (red), and polyethylene (blue) treatments (i.e., linear microplastics are in warm colors: yellow and red, nonlinear microplastics are in cold colors: blue and green).

Considerations about the Apparent Nonmonotonicity of Some Responses. An interesting feature of some of the proxies of soil biophysical environment was an apparent lack of monotonicity in responses to microplastic contamination. In other words, some of the low concentrations of microplastic seem to cause stronger effects than high concentrations compared to the control. Nonmonotonic responses have been conceptualized and empirically observed in complex systems ${ }^{38}$ and soils. ${ }^{39}$ In the following paragraphs we explore in a general context how changes in existing processes, inclusion of new processes, and particulate material interaction are possible drivers of such patterns.

A typical nonmonotonic example is particulate metal pollution in estuaries. At low riverine discharges particulate metal in water tends to increase proportionally to riverine flow due to increased erosion and transport of fine sediments, which are rich in adsorbed metal. ${ }^{40}$ However, the hydrodynamics at higher discharges flushes metals to the sea, which decreases metal pollution. That decrease is intensified by the high flow erosion that transports coarser material, which is poor in adsorbed metal. ${ }^{40}$ In that sense, the change in existing processes (e.g., erosion of fine or coarse sediments), or inclusion of new processes (e.g., hydrodynamic flushing) explains the lack of monotonicity. As mentioned earlier, the addition of the various microplastics to control soils represents a simultaneous change in material, particle type, and particle numbers of the soils. Each of these drivers may affect different processes in soils (e.g., pore space, capillarity, wetting processes, etc.), and their interactions might well yield nonlinearity. In fact, changes in soil texture with linearly increasing clay content are known to trigger nonmonotonic responses in the slope of water retention curves, with resulting nonmonotonic soil physical properties. ${ }^{39}$
In a recent study to address lack of monotonicity in an ecotoxicological context the polydispersivity index, a metric of particle-particle interaction, was shown to behave nonmonotonically. ${ }^{41}$ The behavior of the particles was in turn associated with multiple nonmonotonic biological responses. ${ }^{41}$ To the best of our knowledge, it is not yet possible to fully postulate the soil biophysical effects of interactions between microplastics-microplastics, microplastics-natural matter, and natural matter-natural matter. Notwithstanding, it is sensible to propose that in the control pots there would be comparatively more interactions between natural matter, at lower concentrations microplastic particles would interact more with the natural matter, while at higher concentrations processes influenced by microplastics-microplastics interactions become more relevant. Such change in processes might also elicit nonmonotonicity. In fact, the occurrence of hysteresis, nonmonotonicity, and tipping points are some common characteristics of early warning signals for complex systems under critical transitions. ${ }^{38}$ It is highly likely that the soil responses present some of those complex system features, since the soil environment integrates multiple levels of physical, chemical, and biological processes.

Unfortunately it is not easy to assess nonmonotonic doseresponses in experimental data not designed for that purpose. ${ }^{42}$ Soils are very heterogeneous systems at multiple scales and the current study was a common garden experiment which includes naturally enhanced variability and random noise. Therefore, whereas nonmonotonicity in the responses of soil biophysical distress caused by microplastics are possible, further studies are required to clarify this.

Microplastics Change Microbial Function. The abovereported changes on fundamental properties of soils were associated with changes in microbial activity. No significant relationship between concentration of individual microplastic 
types and microbial activities was observed (Figure 6A). However, a significant relationship irrespective of particle type between microplastic concentration (\% of soil) and microbial activity was observed (Figure 6B; $F=6.14, p<0.05$ ). These results agree with the microplastic-driven increased microbial activity found by Liu et al. $^{43}$ in loess soils contaminated up to $28 \%$ of weight with polypropylene smaller than $180 \mu \mathrm{m}$. However, our study differed in multiple aspects, thus complicating comparisons between both experiments. In our experiment, different microplastics were used and the lower microplastic exposures were strongly influenced by linear microplastics. Therefore, the positive relationship between microplastic concentration and microbial activity might be associated with the overall low response of the latter to the fiber treatments. In fact, the qualitative exposure metrics (Figure 3C) revealed that both, polyacrylic and polyester, fibers presented significantly lower microbial activities than control soils $(F=5.20, p<0.05)$ or those exposed to nonlinear microplastics $(F=10.23, p<0.001$ ) (Figure 6C). Other semiquantitative exposure metrics did not reveal any significant effects with the methods adopted here (Figure 6D-F).

The alterations of the biophysical environment observed in the present study in combination with shifts in microbial activity highlight the potential of microplastic to trigger functional changes in soils. Effects can likely not be reduced to just "microplastic" concentrations, since specific particle properties (linear vs nonlinear, size distributions, polymer, etc.) seem to matter. For instance, water stable aggregates and microbial activity constituting two broadly studied proxies of soil health and function ${ }^{44,45}$ were significantly affected. In our experiment, exposure of soils to the various microplastic types tended to alter this association (Figure 7). For instance, the exposure of soil to polyethylene fragments comparatively
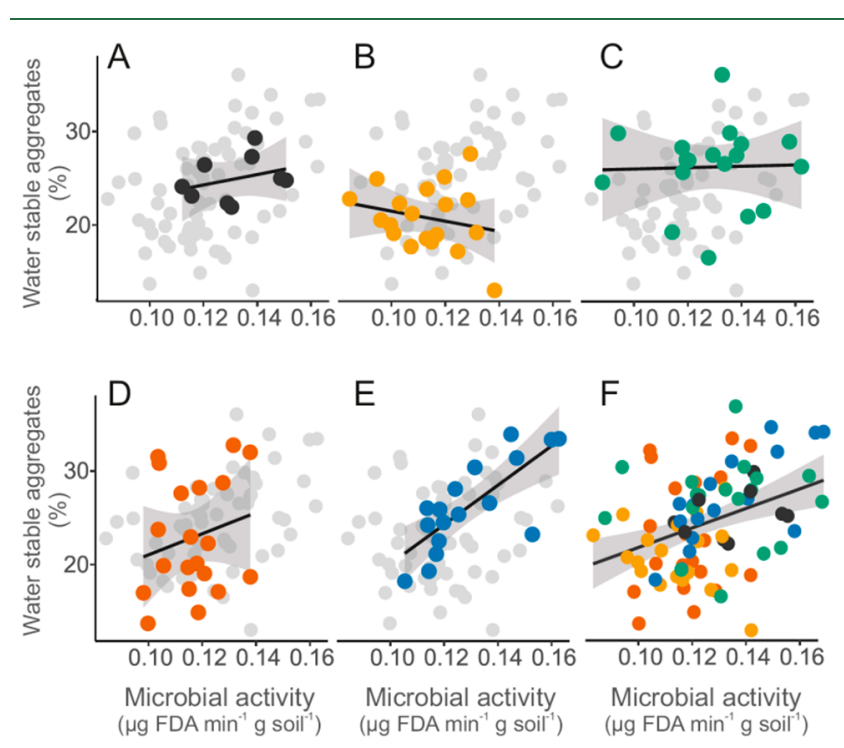

Figure 7. Soil functional relationship between microbial activity and soil aggregation in control (A), or experimentally contaminated soils containing polyacrylic (B), polyamide (C), polyester (D), and polyethylene $(\mathrm{E})$. The general association between these two proxies of soil health is unraveled on panel $\mathrm{F}\left(F=13.01, r^{2}=0.13, p<0.001\right)$. In all panels dark gray, yellow, green, red, and blue colors represent control, polyacrylic, polyamide, polyester, and polyethylene treatments, respectively (i.e., linear microplastics are in warm colors: yellow and red, nonlinear microplastics are in cold colors: blue and green). increased the association between microbial activity and soil aggregation $(F=22.42, p<0.001)$. The contribution of soil biota to soil aggregation has recently been made evident by a meta-analysis that shows a generally positive relationship between these parameters. ${ }^{46}$ This positive association displays considerable variability depending on species investigated, however. ${ }^{46}$ Lehmann et al. ${ }^{46}$ concluded that the diversity and interactions of soil microbes (i.e., bacteria and fungi) are a significant causal factor in soil aggregation. The microbial activity index used here is roughly a measurement of metabolic rate of the entire microbial community, ${ }^{45}$ and it is reasonable to suggest that the altered microbial activities may reflect altered microbial communities. In turn, the changes in the association between microbial activity and water stable aggregates might represent either a shift in microbial community or a modification in the decay of soil organic matter (e.g., preferential electron donor). Disentangling the possible underlying mechanisms of such functional changes is beyond the scope of the present study. Indeed, changes in both, microbial communities and organic matter fate, are conceivable. Liu et al. ${ }^{43}$ suggested that organic matter degradation was accelerated with high concentrations of polypropylene, which resulted in distinct metabolite profiles after 7 and 30 days. Moreover, the fundamental biophysical properties altered by microplastics in our study are known to affect microbial communities. ${ }^{4}$ Therefore, further studies should clarify the mechanisms of the functional responses of soil microbes to the changes in biophysical conditions related to microplastics.

Environmental Implications and Future Directions. The present results highlight the potential of microplastics to alter fundamental properties of the soil biophysical environment with consequences for functional changes in soils. Our findings might have far-reaching consequences for numerous terrestrial ecosystem processes. The current study constitutes the first explorative data-driven analysis of microplastic impacts on soil function. We discuss some of the environmental implications based on the current data, highlight the limitations and suggest future research directions.

To the best of our knowledge, the plastic polymers used in the present experiment are unlikely to undergo relevant degradation, disintegration, or leaching of chemical components within our experimental time scale. ${ }^{37}$ The large microplastic particles seemed intact when observed with the stereomicroscope (Figure 2). Thus, the impacts on soil environment and function might be physical outcomes of the shifts in soil structure, and its subsequent effects on water-soil interaction and microbial function. An analytical quantification of microplastics was not performed here due to a lack of accepted procedures for simultaneously quali-quantitative and nontargeted microplastic analysis, and control soils might therefore already contain detectable amounts of microplastic particles. Nevertheless, microplastic contamination levels simulated here were within the range of contaminated soils near anthropogenically affected landscapes. ${ }^{47}$ Such soil exposure levels are particularly relevant in the context of the near-permanent increasing microplastic pollution, ${ }^{20}$ and because current assessments might underestimate environmental microplastic levels (see SI S1D).

We report significant changes using methods established for the assessment of soil function and health. In this context, it is worth noting that these methods were developed for soils composed mostly of natural particles. It is unclear whether the 
observed changes might reveal increases or decreases in soil health since we used those methods here to determine impacts of microplastic particles that present structural and compositional properties quite distinct from natural soil constituents. For instance, soil bulk density typically correlates with soil physical quality and rootability, ${ }^{39}$ that is, higher bulk density might reduce root growth. However, it is unclear whether the reduction of bulk density by the polyethylene particles (which are lighter than average soils) would have positive consequences for root growth, as it might not necessarily represent increments in soil porosity. Similarly, decreases in water stable aggregates are often regarded as impoverishment of soil structure as it might reduce diversity of soil microenvironments. ${ }^{4,44}$ Both microplastic fibers tested here significantly decreased water stable aggregates (Figure 5) and the fraction of soil forming dry aggregates larger than $1 \mathrm{~mm}$ (SI 1F). Notwithstanding, polyacrylic and polyester microfibers concurrently increased the formation of large soil clumps and therefore potentially provided additional macro-structures absent in the control soil (Figure 2). Therefore, the current data reveal microplastic-driven alterations of soil function, and further studies are required to determine whether such changes result in deleterious environmental consequences.

The most noticeable impacts reported here were associated with the exposure to polyester fibers. This might constitute the first experimental result concerning possible effects on terrestrial systems by one of the commonly produced environmental microplastics. ${ }^{20}$ Polyester fibers account for $\sim 70 \%$ of global plastic fiber production and represent one of the few major plastic contaminants for which there is no relevant recycling. ${ }^{20}$ In this sense, the present study introduces the relevance of a microplastic contaminant ${ }^{8}$ of likely worldwide presence as a potential environmental change factor. Further research is required before this potential can be assessed in a quantitative manner. For instance, most biophysical and functional changes seemed to be associated with idiosyncrasies of microplastic types (e.g., polymer, particle structure, surface oxidation status, and size). Therefore, future comparative studies should look at various soil types, climatic regimes, temporal scales, and microplastic properties to test whether polyester fibers are an important driver of terrestrial global change.

Moreover, the idiosyncratic effects of microplastic types on soil function were not as evident when exposure metrics were assessed on the basis of semiquantitative or qualitative metrics that lump microplastic polymer or particle numbers into a single uniform stressor (Figure 3-6B-C, SI Figure S1 E). This highlights the relevance of simultaneous qualitative and quantitative and nontargeted microplastic analyses for scientifically defensible risk assessment of microplastics on soil. Semiquantitative or only qualitative metrics are currently the most common way of reporting environmental microplastic in both terrestrial and aquatic systems. ${ }^{12,14,48,49}$ Therefore, many of the studies that do not consider the individual microplastic types in a quantitative manner might be of limited value to assess the potential environmental impacts reported here. The term "microplastic" might be useful as a category of contaminants. However, treating all these diverse particles as a uniform anthropogenic stressor in environmental monitoring might significantly compromise environmental risk assessments.

In summary, a solid understanding of the potential impacts of microplastics on terrestrial systems requires special attention to processes taking place within soils. We observed alterations in fundamental properties defining the soil biophysical environment. Such impacts were associated with changes in microbial activity. Further quantitative studies on terrestrial microplastic pollution are required to assess whether this might represent shifts in the diversity of soil microbiota or other deleterious effects on soil health. Our study highlights that microplastics could affect the natural functioning of terrestrial ecosystems in important ways other than eliciting direct lethal toxicity. If extended to other soils and plastic types, the processes unravelled here suggest that microplastics are relevant long-term anthropogenic stressors and drivers of global change in terrestrial ecosystems.

\section{ASSOCIATED CONTENT}

\section{Supporting Information}

The Supporting Information is available free of charge on the ACS Publications website at DOI: 10.1021/acs.est.8b02212.

Data generated during experimental phase of the current study (TXT)

$\mathrm{R}$ script for data visualization and statistical inference (PDF)

Additional figures and information mentioned in the main text (PDF)

\section{AUTHOR INFORMATION}

\section{Corresponding Author}

*E-mail: machadoaas@gmail.com.

ORCID

Anderson Abel de Souza Machado: 0000-0001-6969-7430

Notes

The authors declare no competing financial interest.

\section{ACKNOWLEDGMENTS}

We acknowledge the always helpful laboratory assistance from Sabine Buchert, Erik Faltin, and Wibke Kleiner. We thank Saskia Rehse for providing the polyamide beads. The experimental design and data interpretation were greatly improved by the scientific discussions with Carlos Aguilar, Yudi Lozano, and Diana R. Andrade-Linares. This work was supported by a fellowship of the Dahlem Research School HONORS program funded by Deutsche Forschungsgemeinschaft and the Erasmus Mundus Joint Doctorate Program SMART (Science for the Management of Rivers and their Tidal Systems) funded with the support of the EACEA of the European Union. MR acknowledges support from the ERC Advanced Grant "Gradual Change".

\section{REFERENCES}

(1) Jung, M.; Reichstein, M.; Ciais, P.; Seneviratne, S. I.; Sheffield, J.; Goulden, M. L.; Bonan, G.; Cescatti, A.; Chen, J. Q.; de Jeu, R.; Dolman, A. J.; Eugster, W.; Gerten, D.; Gianelle, D.; Gobron, N.; Heinke, J.; Kimball, J.; Law, B. E.; Montagnani, L.; Mu, Q. Z.; Mueller, B.; Oleson, K.; Papale, D.; Richardson, A. D.; Roupsard, O.; Running, S.; Tomelleri, E.; Viovy, N.; Weber, U.; Williams, C.; Wood, E.; Zaehle, S.; Zhang, K. Recent decline in the global land evapotranspiration trend due to limited moisture supply. Nature 2010, 467 (7318), 951-954.

(2) Schroter, D.; Cramer, W.; Leemans, R.; Prentice, I. C.; Araujo, M. B.; Arnell, N. W.; Bondeau, A.; Bugmann, H.; Carter, T. R.; Gracia, C. A.; de la Vega-Leinert, A. C.; Erhard, M.; Ewert, F.; Glendining, M.; House, J. I.; Kankaanpaa, S.; Klein, R. J. T.; Lavorel, S.; Lindner, M.; Metzger, M. J.; Meyer, J.; Mitchell, T. D.; Reginster, 
I.; Rounsevell, M.; Sabate, S.; Sitch, S.; Smith, B.; Smith, J.; Smith, P.; Sykes, M. T.; Thonicke, K.; Thuiller, W.; Tuck, G.; Zaehle, S.; Zierl, B. Ecosystem service supply and vulnerability to global change in Europe. Science 2005, 310 (5752), 1333-1337.

(3) Bender, S. F.; Wagg, C.; van der Heijden, M. G. A., An Underground Revolution: Biodiversity and Soil Ecological Engineering for Agricultural Sustainability. Trends Ecol. Evol. 201631, (6), $440-452$.

(4) Six, J.; Frey, S. D.; Thiet, R. K.; Batten, K. M. Bacterial and fungal contributions to carbon sequestration in agroecosystems. Soil Sci. Soc. Am. J. 2006, 70 (2), 555-569.

(5) Rillig, M. C. Microplastic in Terrestrial Ecosystems and the Soil? Environ. Sci. Technol. 2012, 46 (12), 6453-6454.

(6) Jambeck, J. R.; Geyer, R.; Wilcox, C.; Siegler, T. R.; Perryman, M.; Andrady, A.; Narayan, R.; Law, K. L. Plastic waste inputs from land into the ocean. Science 2015, 347 (6223), 768-771.

(7) Nizzetto, L.; Futter, M.; Langaas, S. Are Agricultural Soils Dumps for Microplastics of Urban Origin? Environ. Sci. Technol. 2016, 50 (20), 10777-10779.

(8) de Souza Machado, A. A.; Kloas, W.; Zarfl, C.; Hempel, S.; Rillig, M. C. Microplastics as an emerging threat to terrestrial ecosystems. Global Change Biology 2018, 24 (4), 1405-1416.

(9) Ng, E.-L.; Huerta Lwanga, E.; Eldridge, S. M.; Johnston, P.; Hu, H.-W.; Geissen, V.; Chen, D. An overview of microplastic and nanoplastic pollution in agroecosystems. Sci. Total Environ. 2018, 627, $1377-1388$

(10) Steinmetz, Z.; Wollmann, C.; Schaefer, M.; Buchmann, C.; David, J.; Troeger, J.; Munoz, K.; Fror, O.; Schaumann, G. E. Plastic mulching in agriculture. Trading short-term agronomic benefits for long-term soil degradation? Sci. Total Environ. 2016, 550, 690-705.

(11) Nizzetto, L.; Bussi, G.; Futter, M. N.; Butterfield, D.; Whitehead, P. G. A theoretical assessment of microplastic transport in river catchments and their retention by soils and river sediments. Environ. Sci. Process Impacts 2016, 18 (8), 1050-9.

(12) Dris, R.; Gasperi, J.; Saad, M.; Mirande, C.; Tassin, B. Synthetic fibers in atmospheric fallout: A source of microplastics in the environment? Mar. Pollut. Bull. 2016, 104 (1-2), 290-293.

(13) Weithmann, N.; Möller, J. N.; Löder, M. G. J.; Piehl, S.; Laforsch, C.; Freitag, R., Organic fertilizer as a vehicle for the entry of microplastic into the environment. Sci. Adv. 2018, 4, (4).eaap8060

(14) Scheurer, M.; Bigalke, M., Microplastics in swiss floodplain soils. Environ. Sci. Technol. 2018.523591

(15) Huerta Lwanga, E.; Gertsen, H.; Gooren, H.; Peters, P.; Salanki, T.; van der Ploeg, M.; Besseling, E.; Koelmans, A. A.; Geissen, $\mathrm{V}$. Incorporation of microplastics from litter into burrows of Lumbricus terrestris. Environ. Pollut. 2017, 220 (Pt A), 523-531.

(16) Rillig, M. C.; Ziersch, L.; Hempel, S. Microplastic transport in soil by earthworms. Sci. Rep. 2017, 7 (1), 1362.

(17) Maass, S.; Daphi, D.; Lehmann, A.; Rillig, M. C. Transport of microplastics by two collembolan species. Environ. Pollut. 2017, 225, $456-459$.

(18) Zubris, K. A. V.; Richards, B. K. Synthetic fibers as an indicator of land application of sludge. Environ. Pollut. 2005, 138 (2), 201-211.

(19) Rillig, M. C.; Ingraffia, R.; de Souza Machado, A. A. Microplastic Incorporation into Soil in Agroecosystems. Front. Plant Sci. 2017, 8, 1805.

(20) Geyer, R.; Jambeck, J. R.; Law, K. L., Production, use, and fate of all plastics ever made. Science Advances 2017, 3, (7).e1700782

(21) Fuller, S.; Gautam, A. A Procedure for Measuring Microplastics using Pressurized Fluid Extraction. Environ. Sci. Technol. 2016, 50 (11), 5774-80.

(22) Rillig, M. C.; Mardatin, N. F.; Leifheit, E. F.; Antunes, P. M. Mycelium of arbuscular mycorrhizal fungi increases soil water repellency and is sufficient to maintain water-stable soil aggregates. Soil Biol. Biochem. 2010, 42 (7), 1189-1191.

(23) Verbruggen, E.; Jansa, J.; Hammer, E. C.; Rillig, M. C.; Vries, F. Do arbuscular mycorrhizal fungi stabilize litter-derived carbon in soil? J. Ecol. 2016, 104 (1), 261-269.
(24) Blasing, M.; Amelung, W. Plastics in soil: Analytical methods and possible sources. Sci. Total Environ. 2018, 612, 422-435.

(25) Elert, A. M.; Becker, R.; Duemichen, E.; Eisentraut, P.; Falkenhagen, J.; Sturm, H.; Braun, U. Comparison of different methods for MP detection: What can we learn from them, and why asking the right question before measurements matters? Environ. Pollut. 2017, 231, 1256-1264.

(26) Schnurer, J.; Rosswall, T. Fluorescein Diacetate Hydrolysis As a Measure of Total Microbial Activity in Soil and Litter. Environ. Technol. 1982, 43 (6), 1256-1261.

(27) Nijland, R. J. O. H. J., Determining the saturated hydraulic conductivity. In Drainage Principles and Applications, H. P., Ritzema, Ed.; International Institute for Land Reclamation and Improvement (ILRI): Wageningen, The Netherlands, 1994; Vol. 16, p 37.

(28) Kemper, W.D.; R. C. R., Aggregate stability and size distribution. In Methods of Soil Analysis. Part 1. Physical and Mineralogical Properties, Including Statistics of Measurement and Sampling; Klute, E. A., Ed. American Society of Agronomy- Soil Science of America: Madison, WI, 1986; pp 425-442.

(29) Team, R. C. R: A language and environment for statistical computing. In $R$ Foundation for Statistical Computing Vienna, Austria, 2015.

(30) Hadley Wickham, R. F.; Lionel Henry, Kirill Müller RStudio A Grammar of Data Manipulation; CRAN: 2017.

(31) Pinheiro, J.; Bates, D.; Sarkar, D.; R Core Team nlme: Linear and Nonlinear Mixed Effects Models. R package version 3.1-137, https://CRAN.R-project.org/package $=$ nlme, 2018.

(32) Douglas Bates, M. M., Ben Bolker, Steven Walker, Rune Haubo Bojesen Christensen, Henrik Singmann, Bin Dai, Fabian Scheipl, Gabor Grothendieck Peter Green Linear Mixed-Effects Models using 'Eigen' and S4, 1.1-17; CRAN.

(33) Bartón, K. Multi-Model Inference; CRAN: 2018.

(34) Hadley Wickham, W. C. RStudio Create Elegant Data Visualisations Using the Grammar of Graphics, 2.2.1; CRAN: 2016.

(35) Jeffrey B. Arnold, G. D., Bo Werth, Brian Weitzner, Joshua Kunst, Baptise Auguie, Bob Rudis, Hadley Wickham, Justin Talbot Joshua London Extra Themes, Scales and Geoms for 'ggplot2', 3.4.2; CRAN: 2018.

(36) Wilke, C. O. Cowplot: Streamlined Plot Theme and Plot Annotations for 'ggplot2', 0.9.2, 2017.

(37) Lambert, S.; Wagner, M. Environmental performance of biobased and biodegradable plastics: the road ahead. Chem. Soc. Rev. 2017, 46 (22), 6855-6871.

(38) Scheffer, M.; Bascompte, J.; Brock, W. A.; Brovkin, V.; Carpenter, S. R.; Dakos, V.; Held, H.; van Nes, E. H.; Rietkerk, M.; Sugihara, G. Early-warning signals for critical transitions. Nature 2009, $461,53$.

(39) Dexter, A. R. Soil physical quality - Part I. Theory, effects of soil texture, density, and organic matter, and effects on root growth. Geoderma 2004, 120 (3-4), 201-214.

(40) de Souza Machado, A. A.; Spencer, K.; Kloas, W.; Toffolon, M.; Zarfl, C. Metal fate and effects in estuaries: A review and conceptual model for better understanding of toxicity. Sci. Total Environ. 2016, $541,268-281$.

(41) de Souza Machado, A. A.; Zarfl, C.; Rehse, S.; Kloas, W. LowDose Effects: Nonmonotonic Responses for the Toxicity of a Bacillus thuringiensis Biocide to Daphnia magna. Environ. Sci. Technol. 2017, 51 (3), 1679-1686.

(42) Environmental Directorate Joint Meeting of the Chemicals Committee and The Working Party on Chemicals, P., and Biotechnology Current Approaches in the Statistical Analysis of Ecotoxicity Data: A Guidance to Application, 2006.

(43) Liu, H. F.; Yang, X. M.; Liu, G. B.; Liang, C. T.; Xue, S.; Chen, H.; Ritsema, C. J.; Geissen, V. Response of soil dissolved organic matter to microplastic addition in Chinese loess soil. Chemosphere 2017, 185, 907-917.

(44) Zheng, W.; Morris, E. K.; Lehmann, A.; Rillig, M. C. Interplay of soil water repellency, soil aggregation and organic carbon. A metaanalysis. Geoderma 2016, 283, 39-47. 
(45) Green, V. S.; Stott, D. E.; Diack, M. Assay for fluorescein diacetate hydrolytic activity: Optimization for soil samples. Soil Biol. Biochem. 2006, 38 (4), 693-701.

(46) Lehmann, A.; Zheng, W.; Rillig, M. C. Soil biota contributions to soil aggregation. Nature Ecology \& Evolution 2017, 1 (12), 18281835.

(47) Fuller, S.; Gautam, A. A Procedure for Measuring Microplastics using Pressurized Fluid Extraction. Environ. Sci. Technol. 2016, 50 (11), 5774-5780.

(48) Holland, E. R.; Mallory, M. L.; Shutler, D. Plastics and other anthropogenic debris in freshwater birds from Canada. Sci. Total Environ. 2016, 571, 251-258.

(49) Mintenig, S. M.; Int-Veen, I.; Loder, M. G.; Primpke, S.; Gerdts, G. Identification of microplastic in effluents of waste water treatment plants using focal plane array-based micro-Fourier-transform infrared imaging. Water Res. 2017, 108, 365-372. 